\title{
String landscape guide to soft SUSY breaking terms
}

\author{
Howard Baer@, ${ }^{1, *}$ Vernon Barger, ${ }^{2, \dagger}$ Shadman Salam, ${ }^{1, *}$ and Dibyashree Sengupta $\odot^{1, \S}$ \\ ${ }^{1}$ Homer L. Dodge Department of Physics and Astronomy, \\ University of Oklahoma, Norman, Oklahoma 73019, USA \\ ${ }^{2}$ Department of Physics, University of Wisconsin, Madison, Wisconsin 53706, USA
}

(Received 11 June 2020; accepted 14 September 2020; published 12 October 2020)

\begin{abstract}
We examine several issues pertaining to statistical predictivity of the string theory landscape for weak scale supersymmetry (SUSY). We work within a predictive landscape wherein superrenormalizable terms scan while renormalizable terms do not. We require stringy naturalness wherein the likelihood of values for observables is proportional to their frequency within a fertile patch of landscape including the minimal supersymmetric standard model as low energy effective theory with a pocket-universe value for the weak scale nearby to its measured value in our universe. In the string theory landscape, it is reasonable that the soft terms enjoy a statistical power-law draw to large values, subject to the existence of atoms as we know them (atomic principle). We argue that gaugino masses, scalar masses, and trilinear soft terms should each scan independently. In addition, the various scalars should scan independently of each other unless protected by some symmetry. The expected nonuniversality of scalar masses-once regarded as an undesirable feature-emerges as an asset within the context of the string landscape picture. In models such as heterotic compactifications on Calabi-Yau manifolds, where the tree-level gauge kinetic function depends only on the dilaton, gaugino masses may scale mildly, while scalar masses and A terms, which depend on all the moduli, may scale much more strongly leading to a landscape solution to the SUSY flavor and $C P$ problems in spite of nondiagonal Kähler metrics. We present numerical results for Higgs and sparticle mass predictions from the landscape within the generalized mirage mediation SUSY model and discuss resulting consequences for LHC SUSY and weakly interacting massive particles dark matter searches.
\end{abstract}

DOI: 10.1103/PhysRevD.102.075012

\section{INTRODUCTION}

The laws of physics as we know them are beset with several fine-tuning problems that can be interpreted as omissions in our present level of understanding. It is hoped that these gaps may be filled by explanations requiring additional input from physics beyond the Standard Model (SM). One of these, the strong $C P$ problem, is solved via the introduction of a global Peccei-Quinn (PQ) symmetry and its concomitant axion $a$. Another, the gauge hierarchy or Higgs mass problem, is solved via the introduction of weak scale supersymmetry wherein the SM Higgs mass quadratic divergences are rendered instead to be more mild

\footnotetext{
*baer@ou.edu

†barger@pheno.wisc.edu

*shadman.salam@ou.edu

${ }^{\S}$ Dibyashree.Sengupta-1@ou.edu
}

Published by the American Physical Society under the terms of the Creative Commons Attribution 4.0 International license. Further distribution of this work must maintain attribution to the author(s) and the published article's title, journal citation, and DOI. Funded by SCOAP ${ }^{3}$. log divergences. ${ }^{1}$ In this latter case, the nondiscovery of supersymmetry (SUSY) particles at LHC has led to concerns of a little hierarchy problem (LHP), wherein one might expect the weak energy scale $m_{\text {weak }} \sim m_{W, Z, h}$ to be in the multi- $\mathrm{TeV}$ range rather than at its measured value $m_{\text {weak }} \simeq 100 \mathrm{GeV}$. A third fine-tuning problem is the cosmological constant (CC) problem, wherein one expects the cosmological constant $\Lambda \sim m_{P}^{2} \sim 6 \times 10^{54} \mathrm{eV}^{2}$ as opposed to its measured value $\Lambda \simeq 4.33 \times 10^{-66} \mathrm{eV}^{2}$. The most plausible solution to the $\mathrm{CC}$ problem is Weinberg's anthropic solution [2,3]: the value of $\Lambda$ ought to be as natural as possible subject to generating a pocket universe whose expansion rate is not so rapid that structure in the form of galaxy condensation should not occur (this is called the structure principle).

\footnotetext{
${ }^{1}$ The resolution of the gauge hierarchy problem via softly broken supersymmetry is an example of 't Hooft's technical naturalness [1] in that the small weak scale (in relation to the Planck scale) is permissible in that as soft SUSY breaking terms approach zero, the theory becomes more (super)symmetric. In this case, the magnitude of the weak scale is a consequence of supersymmetry breaking [see Eq. (2)].
} 
The anthropic CC solution emerges automatically from the string theory landscape of (metastable) vacua $[4,5]$ wherein each vacuum solution generates a different low energy effective field theory (EFT) and hence apparently different laws of physics (gauge groups, matter content, $\Lambda$, $m_{\text {weak }}$, etc.). A commonly quoted value for the number of flux vacua in IIB string theory is $N_{\text {vac }} \sim 10^{500}$ [6]. If the CC is distributed (somewhat) uniformly across its (anthropic) range of values, then it may not be surprising that we find ourselves in a pocket universe with $\Lambda \sim 10^{-120} m_{P}^{2}$ since if it were much bigger, we would not be here. The situation is not dissimilar to the human species finding itself fortuitously on a moderate size planet a moderate distance from a stable, class-M star: the remaining vast volume of the solar system where we might also find ourselves is inhospitable to liquid water and life as we know it, and we would never have evolved anywhere else.

An essential element to allow Weinberg's reasoning to be predictive is that in the subset of pocket universes with varying cosmological constants, the remaining laws of physics as encoded in the Standard Model stay the same: only $\Lambda$ is scanned by the multiverse. Such a subset ensemble of pocket universes is sometimes referred to as a fertile patch [7]. Arkani-Hamed et al. (ADK) [8] argue that only superrenormalizable Lagrangian terms should scan in the multiverse while renormalizable terms such as gauge and Yukawa couplings will have their values fixed by dynamics. In the case of an ensemble of SM-like pocket universes with the same gauge group and matter content, with Higgs potential given by

$$
V_{\mathrm{SM}}=-\mu_{\mathrm{SM}}^{2} \phi^{\dagger} \phi+\lambda\left(\phi^{\dagger} \phi\right)^{2}
$$

(where $\phi$ is the usual SM Higgs doublet), then just $\mu_{\mathrm{SM}}$ and $\Lambda$ should scan. This would then allow for the possibility of an anthropic solution to the gauge hierarchy problem in that the value of $\mu_{\mathrm{SM}}$ [wherein $m_{h}^{2}$ (tree) $=2 \mu_{\mathrm{SM}}^{2}$ ] would be anthropically selected to cancel off the (regularized) quadratic divergences. Such a scenario is thought to offer an alternative to the usual application of naturalness, which instead would require the advent of new physics at or around the weak scale.

Here, when we refer to naturalness of a physical theory, we refer to

practical naturalness: wherein each independent contribution to any physical observable is required to be comparable to or less than its measured value.

For instance, practical naturalness was successfully applied by Gaillard and Lee to predict the value of the charm quark mass based on contributions to the measured value of the $K_{L}-K_{S}=\Delta m_{K}$ mass difference [9]. In addition, it can be claimed that perturbative calculations in theories such as QED are practically natural (up to some effective theory cutoff $\Lambda_{\mathrm{QED}}$ ). While divergent contributions to observables appear at higher orders, these are dependent quantities: once dependent quantities are combined, higher order contributions to observables are comparable to or less than their measured values. Thus, we understand the concept of practical naturalness and the supposed predictivity of a theory to be closely aligned.

To place the concept of naturalness into the context of the landscape of string theory vacua, Douglas has proposed the notion of stringy naturalness [10]:

stringy naturalness: the value of an observable $\mathrm{O}_{2}$ is more natural than a value $\mathcal{O}_{1}$ if more phenomenologically viable vacua lead to $\mathcal{O}_{2}$ than to $\mathcal{O}_{1}$.

If we apply this definition to the cosmological constant, then phenomenologically viable is interpreted in an anthropic context in that we must veto vacua which do not allow for structure formation (in the form of galaxy condensation). Out of the remaining viable vacua, we would expect $\Lambda$ to be nearly as large as anthropically possible since there is more volume in parameter space for larger values of $\Lambda$. Such reasoning allowed Weinberg to predict the value of $\Lambda$ to within a factor of a few of its measured value more than a decade before its value was determined from experiment [2,3]. The stringy naturalness of the cosmological constant is but one example of what ADK call living dangerously: the values of parameters scanned by the landscape are likely to be selected to be just large enough, but not so large as to violate some fragile feature of the world we live in (in this case the existence of galaxies).

The minimal supersymmetric standard model (MSSM) is touted as a natural solution to the gauge hierarchy problem. This is because in the MSSM log divergent contributions to the weak scale are expected to be comparable to the weak scale for soft SUSY breaking terms $\sim m_{\text {weak }}$. But is the MSSM also more stringy natural than the SM? The answer given in Ref. [11] is yes. For the case of the SM valid up to some energy scale $\Lambda_{\mathrm{SM}} \gg m_{\text {weak }}$, there is only an exceedingly tiny (fine-tuned) range of $\mu_{\mathrm{SM}}^{2}$ values which allow for pocket-universe $m_{\text {weak }}^{\mathrm{PU}} \sim m_{\text {weak }}$ (measured). In contrast, within the MSSM there is a very broad range of superpotential $\mu$ values which allow for $m_{\text {weak }}^{\mathrm{PU}} \sim$ $m_{\text {weak }}$ (measured), provided other contributions to the weak scale are also comparable to $m_{\text {weak }}$ (measured) (as borne out by Figs. 2 and 3 of Ref. [11]). For the MSSM, the pocket universe value of the weak scale is given by

$$
\begin{aligned}
\frac{\left(m_{Z}^{\mathrm{PU}}\right)^{2}}{2}= & \frac{m_{H_{d}}^{2}+\Sigma_{d}^{d}-\left(m_{H_{u}}^{2}+\Sigma_{u}^{u}\right) \tan ^{2} \beta}{\tan ^{2} \beta-1} \\
& -\mu^{2} \simeq-m_{H_{u}}^{2}-\Sigma_{u}^{u}-\mu^{2},
\end{aligned}
$$

where the value of $\mu$ is specified by whatever solution to the SUSY $\mu$ problem is invoked [12]. [Here, $m_{H_{u}}^{2}$ and $m_{H_{d}}^{2}$ are the 
Higgs field soft squared masses, and $\Sigma_{d}^{d}$ and $\Sigma_{u}^{u}$ contain over 40 loop corrections to the weak scale (expressions can be found in the Appendix to Ref. [13])]. Thus, the pocket universe value for the weak scale is determined by the soft SUSY breaking terms and the SUSY preserving $\mu$ parameter. If the landscape of string vacua include as low energy effective theories both the MSSM and the SM, then far more vacua with a natural SUSY EFT should lead to $m_{\text {weak }}^{\mathrm{PU}} \sim$ $m_{\text {weak }}$ (measured) as compared to vacua with the SM EFT where $\Lambda_{\mathrm{SM}} \gg m_{\text {weak }}$. In this vein, unnatural SUSY models such as high scale SUSY where $m_{\text {soft }} \gg m_{\text {weak }}$ should also be rare occurrences on the landscape as compared to natural SUSY.

Douglas has also proposed a functional form for the dependence of the distribution of string theory vacua on the SUSY breaking scale [14]. The form expected for gravity/ moduli mediation is given by

$d N_{\text {vac }}\left(m_{\text {hidden }}^{2}, m_{\text {weak }}, \Lambda\right)=f_{\text {SUSY }} \cdot f_{\text {EWFT }} \cdot f_{\mathrm{CC}} \cdot d m_{\text {hidden }}^{2}$,

where the hidden sector SUSY breaking scale $m_{\text {hidden }}^{4}=$ $\sum_{i}\left|F_{i}\right|^{2}+\frac{1}{2} \sum_{\alpha} D_{\alpha}^{2}$ is a mass scale associated with the hidden sector (and usually in SUGRA-mediated models it is assumed $m_{\text {hidden }} \sim 10^{12} \mathrm{GeV}$ such that the gravitino gets a mass $m_{3 / 2} \sim m_{\text {hidden }}^{2}\left(m_{P}\right)$. Consequently, in gravity mediation the visible sector soft terms $m_{\text {soft }} \sim m_{3 / 2}$. As noted by Susskind [15] and Douglas [6], the scanning of the cosmological constant is effectively independent of the determination of the SUSY breaking scale so that $f_{\mathrm{CC}} \sim \Lambda / m_{\text {string }}^{4}$.

Another key observation from examining flux vacua in IIB string theory is that the SUSY breaking $F_{i}$ and $D_{\alpha}$ terms are likely to be uniformly distributed - in the former case as complex numbers while in the latter case as real numbers. Then one expects the following distribution of supersymmetry breaking scales:

$$
f_{\text {SUSY }}\left(m_{\text {hidden }}^{2}\right) \sim\left(m_{\text {hidden }}^{2}\right)^{2 n_{F}+n_{D}-1},
$$

where $n_{F}$ is the number of $F$-breaking fields and $n_{D}$ is the number of $D$-breaking fields in the hidden sector. Even for the case of just a single $F$-breaking term, one expects a linear statistical draw toward large soft terms; $f_{\text {SUSY }} \sim$ $m_{\text {soft }}^{n}$ where $n=2 n_{F}+n_{D}-1$ and in this case where $n_{F}=1$ and $n_{D}=0$ and then $n=1$. For SUSY breaking contributions from multiple hidden sectors, as typically expected in string theory, $n$ can be much larger, with a consequent stronger pull toward large soft breaking terms.

An initial guess for $f_{\mathrm{EWFT}}$, the (anthropic) fine-tuning factor, was $m_{\text {weak }}^{2} / m_{\text {soft }}^{2}$ which would penalize soft terms which were much bigger than the weak scale. This form is roughly suggested by fine-tuning measures such as $\Delta_{\mathrm{EW}}$ where
$\Delta_{\mathrm{EW}}=\mid$ maximal contribution to RHS of Eq.(2)|/( $\left.m_{Z}^{2} / 2\right)$,

where then $f_{\mathrm{EWFT}} \sim \Delta_{\mathrm{EW}}^{-1}$.

This ansatz fails on several points [16]:

(i) Many soft SUSY breaking choices will land one into charge-or-color breaking (CCB) minima of the electroweak (EW) scalar potential. Such vacua would likely not lead to a livable universe and should be vetoed rather than penalized.

(ii) Other choices for soft terms may not even lead to EW symmetry breaking (EWSB). For instance, if $m_{H_{u}}^{2}(\Lambda)$ is too large, then it will not be driven negative to trigger spontaneous EWSB. These possibilities, labeled as no-EWSB vacua, should also be vetoed.

(iii) In the event of appropriate EWSB minima, then sometimes larger high scale soft terms lead to more natural weak scale soft terms. For instance, 1. if $m_{H_{u}}^{2}(\Lambda)$ is large enough that EWSB is barely broken, then $\mid m_{H_{u}}^{2}$ (weak) $\mid \sim m_{\text {weak }}^{2}$ (see Fig. 3 of Ref. [17]). Likewise, 2. if the trilinear soft breaking term $A_{t}$ is big enough, then there is large top squark mixing and the $\Sigma_{u}^{u}\left(\tilde{t}_{1,2}\right)$ terms enjoy large cancellations, rendering them $\sim m_{\text {weak }}^{2}[13,18]$. The same large $A_{t}$ values lift the Higgs mass $m_{h}$ up to the $125 \mathrm{GeV}$ regime. Also, 3. as first/second generation soft masses are pulled to the tens of $\mathrm{TeV}$ regime, two-loop renormalization group equations (RGE) effects actually suppress third generation soft terms so that SUSY may become more natural [19].

If one assumes a solution to the SUSY $\mu$ problem [12], which fixes the value of $\mu$ so that it can no longer be freely fine-tuned to fix $m_{Z}$ at its measured value, then once the remaining SUSY model soft terms are set, one obtains a pocket-universe value of the weak scale as an output: e.g., $m_{Z}^{\mathrm{PU}} \neq m_{Z}$ (measured). Based on nuclear physics calculations by Agrawal et al. [20,21], a pocket universe value of $m_{\text {weak }}^{\mathrm{PU}}$ which deviates from our measured value by a factor of $2-5$ is likely to lead to an unlivable universe as we understand it. Weak interactions and fusion processes would be highly suppressed, and even complex nuclei could not form. This would be a violation of the atomic principle: that atoms as we know them seem necessary to support observers. This is another example of living dangerously: the pull toward large soft terms tend to pull the value of $m_{\text {weak }}^{\mathrm{PU}}$ up in value, but must stop short of a factor of a few times our measured weak scale lest one jeopardize the existence of atoms as we know them. We will adopt a conservative value that the weak scale should not deviate by more than a factor of 4 from its measured value. This corresponds to a value of the fine-tuning measure $\Delta_{\mathrm{EW}} \lesssim 30$. Thus, for our final form of $f_{\mathrm{EWFT}}$ we will adopt [16] 


$$
f_{\mathrm{EWFT}}=\Theta\left(30-\Delta_{\mathrm{EW}}\right)
$$

while also vetoing CCB and no-EWSB vacua.

The above Eq. (3) has been used to generate statistical distributions for Higgs and sparticle masses as expected from the string theory landscape for various assumed values of $n=0-4$, for assumed gravity-mediation model NUHM3 [16], and also for generalized mirage mediation [22]. For values $n \geq 1$, there is a statistical pull on $m_{h}$ to a peak at $m_{h} \simeq 125 \mathrm{GeV}$ in agreement with the measured value of the Higgs boson mass. Also, for $n \geq 1$, typically the gluino gets pulled to mass values $m_{\tilde{g}} \sim 4 \pm 2 \mathrm{TeV}$, i.e., pulled above LHC mass limits. The lighter top squark is pulled to values $m_{\tilde{t}_{1}} \sim 2 \pm 1 \mathrm{TeV}$ while Higgsinos remain in the $m_{\tilde{\chi}_{1,2}^{0}, \tilde{\chi}_{1}^{ \pm}} \sim 100-350 \mathrm{GeV}$ range. Since gaugino masses are pulled to large values, the neutralino mass gap decreases to $m_{\tilde{\chi}_{2}^{0}}-m_{\tilde{\chi}_{1}^{0}} \sim 3-5 \mathrm{GeV}$, making Higgsino pair production $p p \rightarrow \tilde{\chi}_{1}^{0} \tilde{\chi}_{2}^{0}, \tilde{\chi}_{1}^{ \pm} \tilde{\chi}_{2}^{0}$ very difficult to see at LHC via the soft opposite-sign dilepton signature from $\tilde{\chi}_{2}^{0} \rightarrow \tilde{\chi}_{1}^{0} \ell^{+} \ell^{-}$decay [23]. Thus, this simple statistical model of the string landscape correctly predicts both the mass of the lightest Higgs boson and the fact that LHC sees so far no sign of superparticles. And since first/second generation matter scalars are pulled toward a common upper bound in the $20-40 \mathrm{TeV}$ range, it also predicts only slight violations of flavor-changing neutral current and $C P$ violating processes due to a mixed decoupling/quasidegeneracy solution to the SUSY flavor and $C P$ problems [19].

Our goal in this paper is to investigate several issues of soft SUSY breaking terms relevant for the landscape. The first issue is addressed in Sec. II: which soft terms should scan on the landscape and why? Should the soft terms be correlated and then all scan together? (That is, is only a single random number needed to simulate their scan in the multiverse?) Or should they each scan independently? The second issue is, of the soft terms which ought to scan, should they scan with a common exponent $n$, or are there cases where different soft terms would be drawn more strongly to large values than others? (That is, should different $n$ values apply to different soft terms, depending on the string model?) We address both these issues in Sec. II. Then in Sec. III, we apply what we have learned in Sec. II to examine how stringy natural are different regions of model parameter space, as compared to choosing a common exponent $n$ for all scanning soft terms. Since in the landscape picture many of the soft terms are drawn into the tens-of-TeV range, we expect a comparable value of gravitino mass $m_{3 / 2}$, but with $\mathrm{TeV}$ scale gauginos. In such a case, we expect comparable gravity- and anomalymediated contributions to soft terms so that we present our numerical results within the generalized mirage mediation model $\mathrm{GMM}^{\prime}$ [24]. Some discussion on implications for LHC searches along with overall conclusions are presented in Sec. IV.

\section{SOFT SUSY BREAKING TERMS}

With of order $10^{500}$ metastable vacua solutions in string theory, ${ }^{2}$ it may seem bewildering that one might find the exact one which corresponds to our universe. Such a case might destroy the hoped-for predictability of string theory as pertains to particle physics. A way forward was proposed by Douglas and others [26]: instead, use general stringy arguments to ascertain the likely prior distribution of variable quantities in different pocket-universes within the multiverse. By coupling this with pocket-universe selection criteria (anthropics), one might arrive at statistical predictions from the string theory landscape [27,28]. Indeed, Weinberg was able to do this in the case of the cosmological constant even before knowing about the string theory landscape. In this section, we apply a similar approach not only to the overall SUSY breaking scale [14] but also to the individual soft SUSY breaking terms. This approach avoids the necessity of knowing the precise string vacua which gives rise to our own (pocket) universe. For recent arguments on the prior distribution of the overall SUSY breaking scale from moduli stabilization, see Ref. [29] where Kachru-Kallosh-Linde-Trivedi (KKLT) [30] vacua are found to obey a power-law distribution (as we assume) while large volume scenario [31] vacua obey a log distribution. For arguments on whether KKLT (de Sitter) vacua live within the landscape or the swampland, see, e.g., Ref. [32].

\section{A. String compactification setup}

In string theory, the starting point is the 10/11-dimensional UV complete string theory. One then writes the corresponding 10/11-dimensional effective supergravity (SUGRA) theory by integrating out KK modes and other superheavy states. Compactification of the 10/11-dimensional SUGRA on a Calabi-Yau manifold (to preserve $N=1$ SUSY in the ensuing 4D theory) leads to a 4D SUGRA theory containing visible sector fields plus a plethora (of order hundreds) of gravitationally coupled moduli fields, grouped according to complex structure moduli $U_{j}$ and Kähler moduli $T_{i}$. In accord with Refs. [33,34], we will include the dilaton field $S$ among the set of moduli. In simple IIB string models, the dilaton $S$ and complex structure moduli $U_{j}$ are stabilized by flux while the Kähler moduli are stabilized by various nonperturbative effects [30,35]. In explicit constructions, only one or a few Kähler moduli are assumed [30] while realistically of order $\sim 100$ may be expected. The moduli stabilization allows in principle their many vacuum expectation values (VEVs) to be determined, which then determines the many parameters of the effective theory. For simplicity, here we will assume

\footnotetext{
${ }^{2}$ This number was computed by Denef and Douglas [6] for the case of flux compactifications of IIB string theory. In $F$ theory, far higher numbers $\sim 10^{272,000}$ may be expected [25].
} 
the visible sector fields $C_{\alpha}$ consist of the usual MSSM fields. We will also assume that the moduli $S, T_{i}, U_{j}$ form the hidden sector of the 4D theory and provide the required arena for SUSY breaking. From this framework, we will then draw conclusions as to precisely which soft terms will scan independently within the landscape, and how they are selected for by the power-law formula $f_{\text {SUSY }} \sim m_{\text {soft }}^{n}$. While many insights into moduli stabilization were made for the case of IIB string theory, we expect similar mechanisms to occur for other string models (heterotic, etc.) since the various theories are all related by their duality relations.

\section{B. Soft terms in the low energy EFT}

The 4D, $N=1$ supergravity Lagrangian is determined by just two functions that depend on the chiral superfields $\phi_{M}$ of the model: the real gauge invariant Kähler function $G\left(\phi_{M}, \phi_{M}^{*}\right)=K\left(\phi_{M}, \phi_{M}^{*}\right)+\log \left|W\left(\phi_{M}\right)\right|^{2}$ (with $K$ being the real valued Kähler potential and $W$ the holomorphic superpotential) and the holomorphic gauge kinetic function $f_{a}\left(\phi_{M}\right)$. This is presented in units where the reduced Planck scale $m_{P}=M_{\mathrm{Pl}} / \sqrt{8 \pi}=1$. The chiral superfields of SUGRA $\phi_{M}$ are distinguished according to visible sector fields $C^{\alpha}$ and hidden sector fields $h_{m}$. Following $[33,34,36]$, first we expand the superpotential as a power series in terms of the visible sector fields,

$W=\hat{W}\left(h_{m}\right)+\frac{1}{2} \mu_{\alpha \beta}\left(h_{m}\right) C^{\alpha} C^{\beta}+\frac{1}{6} Y_{\alpha \beta \gamma}\left(h_{m}\right) C^{\alpha} C^{\beta} C^{\gamma}+\cdots$,

while the expansion for the Kähler potential is

$$
\begin{aligned}
K= & \hat{K}\left(h_{m}, h_{m}^{*}\right)+\tilde{K}_{\bar{\alpha}, \beta}\left(h_{m}, h_{m}^{*}\right) C^{* \bar{\alpha}} C^{\beta} \\
& +\left[\frac{1}{2} Z_{\alpha \beta}\left(h_{m}, h_{m}^{*}\right) C^{\alpha} C^{\beta}+\text { H.c. }\right]+\cdots,
\end{aligned}
$$

and where the various coefficients of expansion are to-bedetermined functions of the hidden sector fields $h_{m}$. In the above, Greek indices correspond to visible sector fields while lowercase latin indices correspond to hidden sector fields. Uppercase latin indices correspond to general chiral superfields.

The $F$ part of the scalar potential is given by

$$
V\left(\phi_{M}, \phi_{M}^{*}\right)=e^{G}\left(G_{M} K^{M \bar{N}} G_{\bar{N}}-3\right)=\left(\bar{F}^{\bar{N}} K_{\bar{N} M} F^{M}-3 e^{G}\right) .
$$

If some of the fields $h_{m}$ develops VEVs such that at least one of the auxiliary fields $F^{m}=e^{G / 2} \hat{K}^{m \bar{n}} G_{\bar{n}} \neq 0$, then SUGRA is spontaneously broken. The gravitino gains a mass $m_{3 / 2}=e^{G / 2}$ while soft SUSY breaking terms are generated. The soft terms are obtained from the general 4D, $N=1$ supergravity Lagrangian [37] by replacing the hidden fields $h_{m}$ and their $F_{m}$ terms by their VEVs and then taking the flat limit wherein $m_{P} \rightarrow \infty$ while keeping $m_{3 / 2}$ fixed. One is then left with the low energy EFT which consists of a renormalizable global SUSY Lagrangian augmented by soft SUSY breaking terms.

The canonically normalized gaugino masses are given by

$$
M_{a}=\frac{1}{2}\left(\operatorname{Re} f_{a}\right)^{-1} F^{m} \partial_{m} f_{a} .
$$

The unnormalized Yukawa couplings are given by

$$
Y_{\alpha \beta \gamma}^{\prime}=\frac{\hat{W}^{*}}{|\hat{W}|} e^{\hat{K} / 2} Y_{\alpha \beta \gamma}
$$

while the superpotential $\mu$ term is given by

$$
\mu_{\alpha \beta}^{\prime}=\frac{\hat{W}^{*}}{|\hat{W}|} e^{\hat{K} / 2} \mu_{\alpha \beta}+m_{3 / 2} Z_{\alpha \beta}-\bar{F}^{\bar{m}} \partial_{\bar{m}} Z_{\alpha \beta}
$$

The scalar potential is expanded as

$V_{\mathrm{soft}}=m_{\bar{\alpha} \beta}^{\prime 2} C^{* \bar{\alpha}} C^{\beta}+\left(\frac{1}{6} A_{\alpha \beta \gamma}^{\prime} C^{\alpha} C^{\beta} C^{\gamma}+\frac{1}{2} B_{\alpha \beta}^{\prime} C^{\alpha} C^{\beta}+\right.$ H.c. $)$

with unnormalized soft terms given by

$$
\begin{aligned}
m_{\bar{\alpha} \beta}^{\prime 2}= & \left(m_{3 / 2}^{2}+V_{0}\right) \tilde{K}_{\bar{\alpha} \beta}-\bar{F}^{\bar{m}} \\
& \times\left(\partial_{\bar{m}} \partial_{n} \tilde{K}_{\bar{\alpha} \beta}-\partial_{\bar{m}} \tilde{K}_{\bar{\alpha} \gamma} \tilde{K}^{\gamma \bar{\delta}} \partial_{n} \tilde{K}_{\bar{\delta} \beta}\right) F^{n}
\end{aligned}
$$

and

$$
\begin{aligned}
A_{\alpha \beta \gamma}^{\prime}= & \frac{\hat{W}^{*}}{|\hat{W}|} e^{\hat{K} / 2} F^{m}\left[\hat{K}_{m} Y_{\alpha \beta \gamma}+\partial_{m} Y_{\alpha \beta \gamma}\right. \\
& \left.-\left(\tilde{K}^{\delta \bar{\rho}} \partial_{m} \tilde{K}_{\bar{\rho} \alpha} Y_{\delta \beta \gamma}+(\alpha \leftrightarrow \beta)+(\alpha \leftrightarrow \gamma)\right)\right] .
\end{aligned}
$$

We shall not need the (rather lengthy) expression for $B_{\alpha \beta}^{\prime}$.

\section{Implications for the landscape}

\section{Gaugino masses}

The normalized gaugino mass soft terms are given in Eq. (10) where $\operatorname{Re}\left(f_{a}\right)=1 / g_{a}^{2}$. For nonzero gaugino masses, the gauge kinetic function $f_{a}$ must be a nontrivial function of the moduli fields. In most 4D string constructs, $f_{a}$ is then taken as $k_{a} S$ where $k_{a}$ is the Kac-Moody level of the gauge factor. This form of the gauge kinetic function leads to universal gaugino masses which require SUSY breaking in the dilaton field $S$. The remaining moduli can enter $f_{a}$ at the loop level and lead to nonuniversal gaugino masses. If the moduli contribution to $M_{a}$ is comparable to the dilaton contribution, then one might expect nonuniversal gaugino masses, but otherwise the nonuniversality would be a small effect. If the gaugino masses are 
dominantly from the dilaton, then only a single hidden sector field contributes. In this case, one would expect the $f_{\text {SusY }}$ function to scan as $m_{\text {soft }}^{1}$, i.e., a linear scan for the gaugino masses. In Sec. III, we will see that the landscape actually prefers gaugino masses which are suppressed compared to scalar masses: $F^{S} \ll F^{m}$, where $F^{m}$ corresponds to the collective SUSY breaking scale from all the moduli fields. In this case, the loop-suppressed modulimediated terms may be comparable to the dilaton-mediated contribution and nonuniversality might be expected. Also, even if moduli-mediated contributions are small, the anomaly-mediated contributions can be comparable to the universal contribution. To account for this, in Sec. III we will work within the generalized [24] mirage mediation [38] scheme for soft term masses, and we would indeed expect some substantial nonuniversality of gaugino masses. This type of nonuniversality leads to gaugino mass unification at the mirage scale $\mu_{\text {mir }}$ which can be much less than the grand unified theory (GUT) scale $m_{\mathrm{GUT}} \simeq 2 \times 10^{16} \mathrm{GeV}$ where gauge couplings unify. Furthermore, in compactification schemes where the moduli-mediated contribution to $M_{a}$ is comparable to the dilaton contribution, one might expect the gaugino masses to scan as $m_{\text {soft }}^{n}$, where the precise value of $n$ depends on how many moduli fields contribute to the gaugino masses. Since here we are considering that gaugino masses should scan independently of other soft terms, we will denote their $n$ value in $f_{\text {SUSY }}$ hereafter as $n_{1 / 2}$.

\section{Soft scalar masses}

The soft SUSY breaking scalar masses come from Eq. (14). In that equation, the first part, upon normalizing to obtain canonical kinetic terms, leads to diagonal and universal scalar masses. In past times, this was a feature to be sought after since it offered a universality solution to the SUSY flavor problem [39]. The second term involving partial derivatives of the visible sector Kähler metric, leads to nonuniversal soft terms. In particular, we would expect nonuniversal soft scalar masses for the two Higgs doublets $m_{H_{u}}^{2}$ and $m_{H_{d}}^{2}$, along with nonuniversal masses $m_{0}(1), m_{0}(2)$, and $m_{0}(3)$ for each of the generations. Intragenerational universality might be expected to occur, for instance, where $S O(10)$ gauge symmetry survives the compactification (as occurs, for instance, in some orbifold compactification scenarios [40] which lead to local grand unification [41,42]). Then all 16 fields of each generation which fill out the 16-dimensional spinor of $S O(10)$ would have a common mass $m_{0}(i)$ for $i=1-3$. Nonuniversal soft SUSY breaking scalar masses lately are a desired feature in SUGRA models since they allow for radiatively driven naturalness (RNS) [13,18], wherein radiative corrections (via RG equations) drive large high scale soft terms to weak scale values such that the contributions in Eq. (2) to the weak scale are of natural magnitudes. The RNS scenario has a natural home in the string landscape [17]. For instance, if $m_{H_{u}}^{2}$ is statistically favored as large as possible, then instead of being driven to large negative multi-TeV values during the radiative breaking of $S U(2)_{L} \times U(1)_{Y}$ symmetry [43-49], it will be driven to small weak scale values, just barely breaking EW symmetry. This is an example of living dangerously [8] in the string theory landscape, since if the high scale value of $m_{H_{u}}^{2}$ were much bigger, then EW symmetry would not even break.

As mentioned, the expected nonuniversality of soft SUSY scalar masses for each generation in gravity mediation was vexing for many years [39] and, in fact, provided strong motivation for flavor-independent mediation schemes such as gauge mediation [50,51] and anomaly mediation [52-55]. ${ }^{3}$ The original incarnations of these models are highly disfavored, if not ruled out, due to the rather large value of the Higgs mass $m_{h} \simeq 125 \mathrm{GeV}$ [57-59]. Happily, the string theory landscape offers its own solution to both the SUSY flavor and $C P$ problems arising from nonuniversal generations [19]. In the landscape, the statistical selection of soft SUSY breaking scalar masses pulls them to as large of values as possible such that their contributions to the weak scale remain of order the weak scale. The top squark contributions to the weak scale are proportional to the top quark Yukawa couplings, so these soft terms are pulled into the few $\mathrm{TeV}$ regime. However, first and second generation sfermions have much smaller Yukawa couplings and so are pulled much higher, into the $20-40 \mathrm{TeV}$ regime. In fact, the upper bounds on first/second generation sfermions come from two-loop renormalization group (RG) effects which push third generation soft masses smaller [thus aiding naturalness by suppressing $\Sigma_{u}^{u}\left(\tilde{t}_{1,2}\right)$ terms] and then ultimately toward tachyonic. From this effect, the anthropic upper bound is the same for both first and second generation sfermions: they are pulled to large values, but to a common upper bound. This provides a quasidegenerate, decoupling solution to the SUSY flavor and $C P$ problems [19].

Overall, all the SUSY breaking moduli fields should contribute to the soft SUSY breaking scalar masses. Thus, we would expect a landscape selection for scalar masses according to $m_{\mathrm{soft}}^{2 n_{F}+n_{D}-1}$ and thus perhaps a stronger pull on scalar masses to large values than might occur for gauginos. To allow for this effect, we hereafter denote the value of $n$ contributing to selection of soft scalar masses as $n_{0}$.

\section{Trilinears}

The trilinear soft breaking terms, so-called $A$ terms, are given in Eq. (15). These terms again receive contributions from all the SUSY breaking moduli fields and are of order

\footnotetext{
${ }^{3}$ A generalized version of anomaly-mediated SUSY breaking (AMSB) has been proposed [56] which allows for bulk $A$ terms and nonuniversal bulk scalar masses. This version of AMSB allows for $m_{h} \sim 125 \mathrm{GeV}$ and naturalness under the $\Delta_{\mathrm{EW}}$ measure. While winos are still the lightest gauginos, the Higgsinos are the lightest electroweakinos.
} 
$m_{\text {soft }}$. They should scan in the landscape according to $f_{\text {SUSY }} \sim m_{\text {soft }}^{n_{0}}$, similar to the scalar masses. It is worth noting that in Eq. (15) the Yukawa couplings do not in general factor out of the soft terms.

The statistical selection of large $A$ terms pulls the stop mass matrix to maximal mixing; this maximizes the light Higgs mass and hence lifts $m_{h} \rightarrow 125 \mathrm{GeV}[18,60]$. Meanwhile, it also leads to cancellations in the loop contributions to the EW scale $\Sigma_{u}^{u}\left(\tilde{t}_{1}\right)$ and $\Sigma_{u}^{u}\left(\tilde{t}_{2}\right)$, thus decreasing their contributions to the weak scale. For even larger negative values of $A$ parameters, the $\Sigma_{u}^{u}\left(\tilde{t}_{1,2}\right)$ contributions to $m_{\text {weak }}$ increase well beyond $4 m_{\text {weak }}$ (measured) just before pushing top squark soft terms tachyonic leading to CCB minima of the scalar potential [11]. This is another example of living dangerously.

\section{4. $\mu$ parameter}

The bilinear mass term $\frac{1}{2} \mu_{\alpha \beta}\left(h_{m}\right) C^{\alpha} C^{\beta}$ in Eq. (7) is forbidden for almost all matter superfields of the MSSM by gauge invariance. The exception occurs for the vectorlike pair of Higgs doublets $\mu H_{u} H_{d}$ which contain opposite hypercharge assignments, making this an allowed term. Naively, since the term is supersymmetry preserving, one might expect $\mu \sim m_{P}$; on the other hand, due to the scale invariance of string theory, no mass terms are allowed for massless states and one gets $\mu=0$ [61]. Phenomenologically, such a term with $\mu \sim m_{\text {weak }}$ is necessary for appropriate EW symmetry breaking. The conflict among the above issues forms the SUSY $\mu$ problem. ${ }^{4}$ Notice that if $\mu \sim m_{\text {weak }}$ in accord with naturalness, but $m_{\text {soft }} \gtrsim \mathrm{TeV}$ scale, then $\mu \ll m_{\text {soft }}$ and the $\mu$ parameter is also intimately involved in the little hierarchy (LH) problem: why is there a gap opening up between the weak scale and the soft breaking scale? The landscape automatically generates such a LH by pulling soft terms to such large values that EW symmetry is barely broken.

The analysis of soft SUSY breaking terms already contains within it two possible resolutions of the $\mu$ problem, which could be acting simultaneously. These resolutions depend on the mixing between observable sector fields $H_{u}$ and $H_{d}$ with hidden sector fields $h_{m}$. If a value of $Z_{\alpha \beta} \sim$ $\lambda h_{m} / m_{P}$ gains a value $\lambda m_{\text {hidden }}^{2} / m_{P}$ under SUSY breaking, then a $\mu$ parameter or order $m_{\text {soft }}$ is generated [62].

Alternatively, in Eq. (7) where $\mu_{\alpha \beta}$ is a function of hidden sector fields $h_{m}$, if the hidden fields develop a suitable VEV, then a $\mu$ parameter will be generated. In the NMSSM [63], a singlet superfield $X$ is added to the visible sector, and when $X$ obtains a weak scale VEV, then a $\mu$ term is generated. If $\mu_{\alpha \beta}$ contains nonrenormalizable terms such as $\lambda_{\mu} X^{2} / m_{P}$, then upon SUSY breaking a $\mu \sim \lambda_{\mu} m_{\text {hidden }}^{2} / m_{P}$ is developed with $\mu \sim m_{\text {weak }}-m_{\text {soft }}$. This is the Kim-Nilles (KN) mechanism, which originally relied on a PQ

\footnotetext{
${ }^{4}$ Twenty solutions to the SUSY $\mu$ problem are reviewed in Ref. [12].
}

symmetry to forbid the initial $\mu \sim m_{P}$ term. An attractive feature of this approach is that the PQ symmetry is also used to solve the strong $C P$ problem via the supersymmetrized [64-66] Dine-Fischler-Srednicki-Zhitnitski (DFSZ) axion $[67,68]$.

A less attractive feature is that the global PQ symmetry is not compatible with gravity/string theory [69-72]. A way forward is to invoke instead either a (gravity-compatible) discrete gauge symmetry [73] $\mathbb{Z}_{N}$ or a discrete $R$-symmetry $\mathbb{Z}_{N}^{R}$, where the latter might originate as a discrete remnant from 10D Lorentz symmetry breaking after compactification. Then the global PQ symmetry emerges as an accidental, approximate symmetry as a consequence of the underlying discrete gauge or $R$ symmetry. In the latter case, a variety of $\mathbb{Z}_{N}^{R}$ symmetries have been shown to be anomaly-free and consistent with grand unification [74] for $N=4,6,8,12$, and 24 . The largest of these, $\mathbb{Z}_{24}^{R}$, is strong enough to suppress nonrenormalizable contributions to the scalar potential up to powers of $\left(1 / m_{P}\right)^{8}$, which is enough to solve the strong $C P$ problem while maintaining the strong $C P$ angle $\bar{\theta} \lesssim 10^{-10}$. Such an approach is attractive since it solves the strong $C P$ problem, solves the SUSY $\mu$ problem, provides a mechanism for $R$-parity conservation, and suppresses otherwise dangerous dimensionfive proton decay operators [75].

\section{RESULTS FOR GENERALIZED MIRAGE MEDIATION MODEL GMM'}

\section{A. $\mathbf{G M M}^{\prime}$ model and parameter space}

The mirage mediation model is based on comparable moduli- and anomaly-mediated contributions to soft SUSY breaking terms. The boundary conditions are implemented at energy scale $Q=m_{\mathrm{GUT}} \simeq 2 \times 10^{16} \mathrm{GeV}$ where the gauge couplings unify. Under this supposition, the gaugino masses receive a universal moduli-mediated contribution along with an anomaly-mediated contribution which depends on the gauge group beta functions. The offset from universality is compensated for by RGE running to lower mass scales which causes the gaugino masses to unify at the mirage scale $\mu_{\mathrm{mir}}=m_{\mathrm{GUT}} e^{-8 \pi^{2} / \alpha}$ where $\alpha$ parametrizes the relative moduli- to anomaly-mediated contributions to the soft terms. For $\alpha \rightarrow 0$, one recovers pure AMSB while as $\alpha \rightarrow \infty$ dominant moduli mediation is recovered. The smoking gun signature of mirage mediation is that gaugino masses unify at the intermediate mirage scale rather than $m_{\mathrm{GUT}}$. This feature can be tested at $e^{+} e^{-}$ colliders operating at $\sqrt{s}>2 m($ Higg sino $o$ [76,77].

Expressions for the soft SUSY breaking terms have been calculated in Refs. [78-81] under the assumption of simple compactifications of IIB string theory with a single Kähler modulus. For more realistic compactifications with many Kähler moduli, the discrete-valued modular weights are generalized to be continuous parameters in the generalized 
mirage mediation model $\left(\mathrm{GMM}^{\prime}\right)$ [24] which we adopt here.

For the GMM' model, the soft SUSY breaking terms are given by

$$
\begin{gathered}
M_{a}=\left(\alpha+b_{a} g_{a}^{2}\right) m_{3 / 2} / 16 \pi^{2}, \\
A_{\tau}=\left(-a_{3} \alpha+\gamma_{L_{3}}+\gamma_{H_{d}}+\gamma_{E_{3}}\right) m_{3 / 2} / 16 \pi^{2}, \\
A_{b}=\left(-a_{3} \alpha+\gamma_{Q_{3}}+\gamma_{H_{d}}+\gamma_{D_{3}}\right) m_{3 / 2} / 16 \pi^{2}, \\
A_{t}=\left(-a_{3} \alpha+\gamma_{Q_{3}}+\gamma_{H_{u}}+\gamma_{U_{3}}\right) m_{3 / 2} / 16 \pi^{2}, \\
m_{i}^{2}(1,2)=\left(c_{m} \alpha^{2}+4 \alpha \xi_{i}-\dot{\gamma}_{i}\right)\left(m_{3 / 2} / 16 \pi^{2}\right)^{2}, \\
m_{j}^{2}(3)=\left(c_{m 3} \alpha^{2}+4 \alpha \xi_{j}-\dot{\gamma}_{j}\right)\left(m_{3 / 2} / 16 \pi^{2}\right)^{2}, \\
m_{H_{u}}^{2}=\left(c_{H_{u}} \alpha^{2}+4 \alpha \xi_{H_{u}}-\dot{\gamma}_{H_{u}}\right)\left(m_{3 / 2} / 16 \pi^{2}\right)^{2}, \\
m_{H_{d}}^{2}=\left(c_{H_{d}} \alpha^{2}+4 \alpha \xi_{H_{d}}-\dot{\gamma}_{H_{d}}\right)\left(m_{3 / 2} / 16 \pi^{2}\right)^{2} .
\end{gathered}
$$

In the above expressions, the index $i$ runs over the first/ second generation MSSM scalars $i=Q_{1,2}, U_{1,2}, D_{1,2}, L_{1,2}$, and $E_{1,2}$ while $j$ runs overs third generation scalars $j=Q_{3}, U_{3}, D_{3}, L_{3}$, and $E_{3}$. Here, we adopt an independent value $c_{m}$ for the first two matter-scalar generations while the parameter $c_{m 3}$ applies to third generation matter scalars. The independent values of $c_{H_{u}}$ and $c_{H_{d}}$, which set the modulimediated contribution to the Higgs mass-squared soft terms, may conveniently be traded for weak scale values of $\mu$ and $m_{A}$ as is done in the two-parameter nonuniversal Higgs model (NUHM2) [82-87]. This procedure allows for more direct exploration of stringy natural SUSY parameter space where most landscape solutions require $\mu \sim 100-360 \mathrm{GeV}$ in anthropically allowed pocket universes [11]. Thus, the $\mathrm{GMM}^{\prime}$ parameter space is given by

$$
\alpha, m_{3 / 2}, c_{m}, c_{m 3}, a_{3}, \tan \beta, \mu, m_{A}\left(\mathrm{GMM}^{\prime}\right) .
$$

The natural GMM and $\mathrm{GMM}^{\prime}$ models have been incorporated into the event generator program ISAJET7.88 [88] which we use here for spectra generation. (The GMM and $\mathrm{GMM}^{\prime}$ models are equivalent: GMM uses high scale Higgs soft terms $m_{H_{u}}^{2}$ and $m_{H_{d}}^{2}$ parameter choices while GMM' trades these for the more convenient weak scale parameters $\mu$ and $m_{A}$.)

\section{B. Results in the $m_{0}^{M M}$ vs $m_{1 / 2}^{M M}$ plane}

A panoramic view of some of our main results is conveniently displayed in the $m_{0}^{M M}$ vs $m_{1 / 2}^{M M}$ plane which is then analogous to the $m_{0}$ vs $m_{1 / 2}$ plane of the mSUGRA/ CMSSM or NUHM2,3 models. Here, we define $m_{0}^{M M}=$ $\sqrt{c_{m}} \alpha\left(m_{3 / 2} / 16 \pi^{2}\right)$ which is the pure moduli-mediated contribution to scalar masses. The moduli-mediated contribution to gaugino masses is correspondingly given by $m_{1 / 2}^{M M} \equiv \alpha m_{3 / 2} /\left(16 \pi^{2}\right)$.

In Fig. 1(a), we show the $m_{0}^{M M}$ vs $m_{1 / 2}^{M M}$ plane for the case of an $n_{1 / 2}=n_{0}=1$ landscape draw but with $a_{3}=1.6 \sqrt{c_{m}}$, with $c_{m}=c_{m 3}$ and with $\tan \beta=10, m_{A}=2 \mathrm{TeV}$, and $\mu=200 \mathrm{GeV}$. The lower-left yellow region shows where $m_{\tilde{\chi}_{1}^{ \pm}}<103.5 \mathrm{GeV}$ in violation of LEP2 constraints. Also, the lower-left orange box shows where $\Delta_{B G}<30$ (old naturalness calculation). The bulk of the low $m_{1 / 2}$ region here leads to tachyonic top-squark soft terms owing to the large trilinear terms $A_{0}^{M M} \equiv-a_{3} \alpha\left(m_{3 / 2} / 16 \pi^{2}\right)$. This region is nearly flat with increasing $m_{0}$ mainly because the larger we make the GUT scale top-squark squared mass soft terms, the larger is the canceling correction from RG running. For larger $m_{1 / 2}^{M M}$ values, we obtain viable EW vacua since large values of $M_{3}$ help to enhance top squark squared mass running to large positive values. The dots show the expected statistical result of scanning the landscape, and the larger density of dots on the plot corresponds to greater stringy naturalness. We also show the magenta contour of $m_{\tilde{g}}=2.25 \mathrm{TeV}$, below which is excluded by LHC gluino pair searches $[89,90]$. We also show contours of $m_{h}=123$ and $125 \mathrm{GeV}$. The green points are consistent with LHC sparticle search limits and the Higgs mass measurement. From the plot, we see that much of the region of high stringy naturalness tends to lie safely beyond LHC sparticle search limits while at the same time yielding a Higgs mass $m_{h} \simeq 125 \mathrm{GeV}$. While early naturalness calculations preferred low $m_{0}$ and $m_{1 / 2}$ regions [91-94], we see now that stringy naturalness prefers the opposite [11]: as large as possible values of $m_{0}^{M M}$ and $m_{1 / 2}^{M M}$ subject to the (anthropic) condition that $m_{\text {weak }}^{\mathrm{PU}}$ is within a factor of 4 of our measured value (lest the atomic principle be violated). Thus, the most stringy natural region statistically prefers a light Higgs mass $m_{h} \simeq 125 \mathrm{GeV}$ with sparticles beyond LHC Run 2 reach.

In frame Fig. 1(b), we increase the value of $n_{0}$ to 2 while keeping $n_{1 / 2}$ fixed at 1 . Likewise, in frames 1 (c) and 1(d), we increase $n_{0}$ to 3 and 4 , respectively. The number of dots in the various frames are normalized to $\sim 1500$ so that the relative density, indicating the relative stringy natural regions, can be compared on an equal footing. As $n_{0}$ increases, corresponding to more moduli fields contributing to SUSY breaking in the scalar sector, the stringy natural region migrates toward higher values of $m_{0}^{M M}$ and a sharpening of the Higgs mass prediction that $m_{h} \simeq 125 \mathrm{GeV}$. In fact, in frame 1 (d) for $n_{0}=4$, there are only a few scan points with $m_{h}<123 \mathrm{GeV}$. An anti-intuitive conclusion from our calculations is that a $3 \mathrm{TeV}$ gluino is more stringy natural than a $300 \mathrm{GeV}$ gluino.

In Fig. 2, we show the histograms of Higgs mass probability for $n_{1 / 2}=1$ with $n_{0}=1,2,3$, and 4 . As seen from the plot, as $n_{0}$ increases, the probability distribution $d P / d m_{h}$ does indeed sharpen around the value of $m_{h} \simeq 125 \mathrm{GeV}$. 

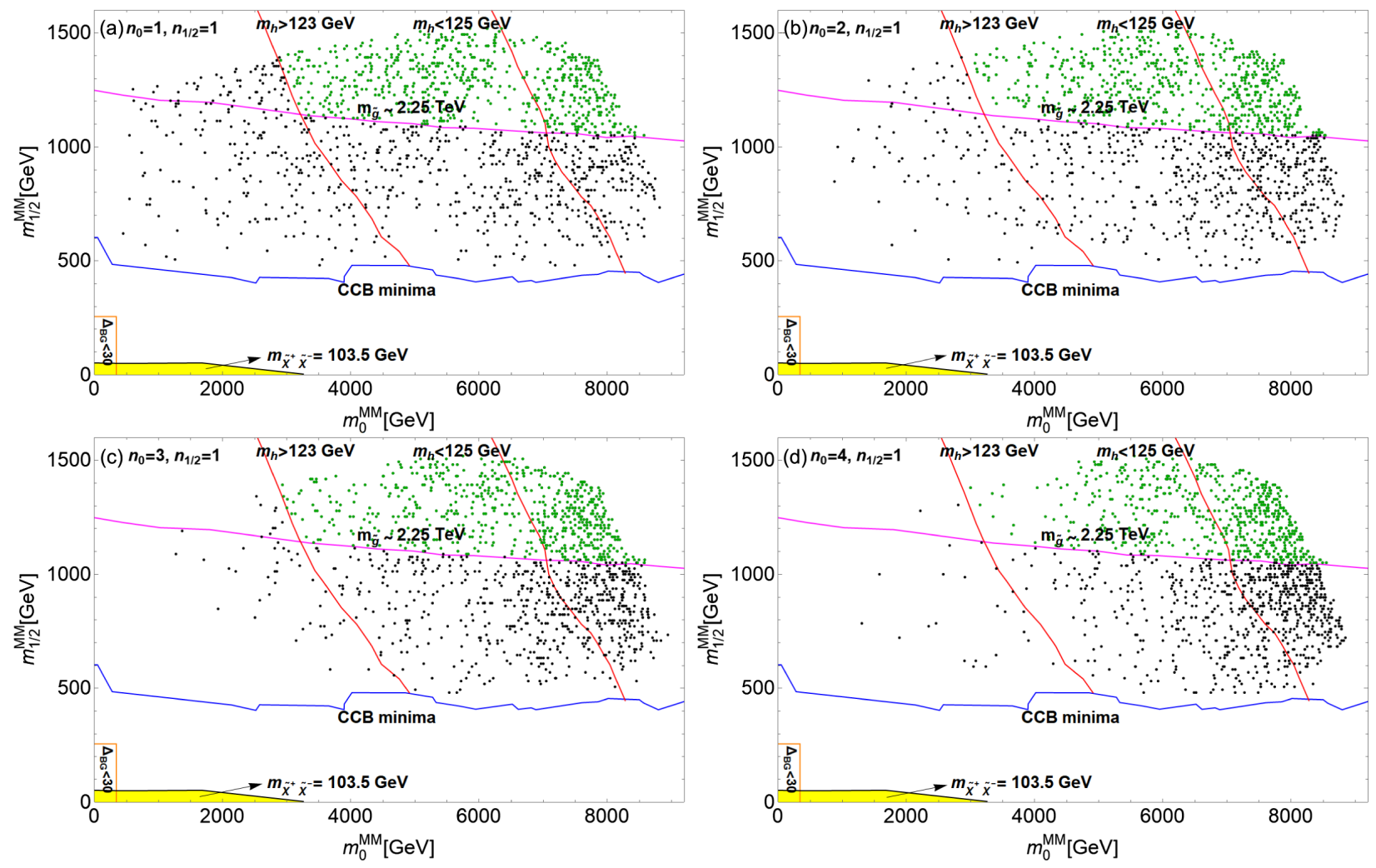

FIG. 1. The $m_{0}^{M M}$ vs $m_{1 / 2}^{M M}$ plane of the GMM' model for a value of $n_{1 / 2}=1$ for all frames but with (a) $n_{0}=1$, (b) $n_{0}=2$, (c) $n_{0}=3$, and (d) $n_{0}=4$. For all frames, we take $m_{3 / 2}=20 \mathrm{TeV}, \mu=200 \mathrm{GeV}, m_{A}=2 \mathrm{TeV}, \tan \beta=10$, and $a_{3}=1.6 \sqrt{c_{m}}$. We require $m_{Z}^{\mathrm{PU}}<4 m_{Z}^{\mathrm{OU}}$.

\section{Parameter space scan procedure for $\mathbf{G M M}^{\prime}$ on the landscape}

We use ISAJET to scan the $\mathrm{GMM}^{\prime}$ model parameter space as follows:

(i) We select a particular value of $m_{3 / 2}=20 \mathrm{TeV}$ which then fixes the AMSB contributions to soft SUSY breaking terms.

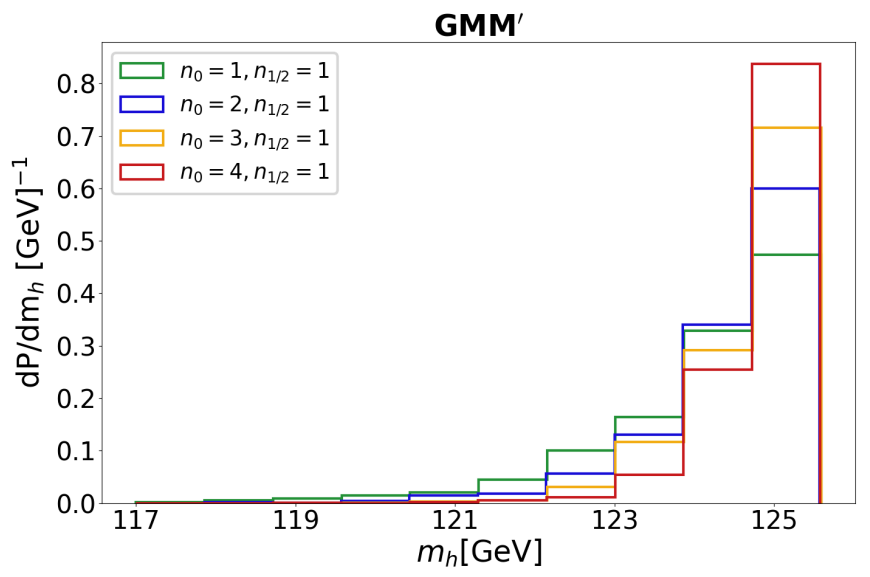

FIG. 2. Probability distribution $d P / d m_{h}$ vs $m_{h}$ for $n_{1 / 2}=1$ and $n_{0}=1,2,3$, and 4 for scans of the $\mathrm{GMM}^{\prime}$ model for $m_{3 / 2}=20 \mathrm{TeV}, \mu=200 \mathrm{GeV}, m_{A}=2 \mathrm{TeV}, \tan \beta=10$, and $a_{3}=1.6 \sqrt{c_{m}}$. We require $m_{Z}^{\mathrm{PU}}<4 m_{Z}^{\mathrm{OU}}$. (ii) We also fix $\mu=200 \mathrm{GeV}$ for a natural solution to the SUSY $\mu$ problem. This then allows for arbitrary values of $m_{Z}^{\mathrm{PU}}$ to be generated but disallows any possibility of fine-tuning $\mu$ to gain the measured value of $m_{Z}^{\mathrm{OU}}$ in our universe.

Next, we will invoke Douglas' power-law selection $[8,14,15]$ of moduli-mediated soft terms relative to AMSB contributions within the $\mathrm{GMM}^{\prime}$ model. Thus, for an assumed value of $n_{1 / 2}$ and $n_{0}$, we will generate

(i) $\alpha^{n_{1 / 2}}$ with $\alpha: 3-25$, corresponding to a power-law statistical selection for moduli/dilaton-mediated gaugino masses $M_{a}(a=1-3$ over the gauge groups).

(ii) $\left(a_{3} \alpha\right)^{n_{0}}$, a power-law statistical selection of modulimediated $A$-terms, with $\left(a_{3} \alpha\right): 3-100$.

(iii) $\left(\sqrt{c_{m 3} \alpha^{2}}\right)^{n_{0}}$ to gain a power-law statistical selection on third generation scalar masses $m_{0}(3)$, with $\left(\sqrt{c_{m 3} \alpha^{2}}\right): 3-80$.

(iv) $\left(\sqrt{c_{m} \alpha^{2}}\right)^{n_{0}}$ to gain a power-law statistical selection on first/second generation scalar masses $m_{0}(1,2)$, with $\left(\sqrt{c_{m} \alpha^{2}}\right): \sqrt{c_{m 3} \alpha^{2}}-320$.

(v) a power-law statistical selection on $m_{H_{d}}^{2}$ via $m_{A}^{n_{0}}$ with $m_{A}: 300-10,000 \mathrm{GeV}$.

(vi) a uniform selection on $\tan \beta$ : 3-50.

We adopt a uniform selection on $\tan \beta$ since this parameter is not a soft term. Note that with this procedure-while 


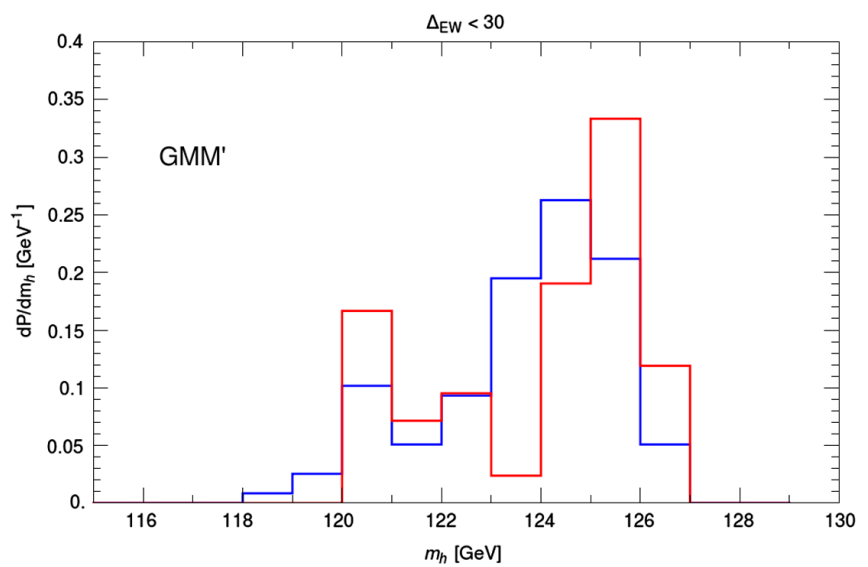

FIG. 3. Probability distribution for mass of light Higgs boson $m_{h}$ for $n_{1 / 2}=1$ with $n_{0}=1$ (blue line) and $n_{0}=2$ (red line) from statistical scans over the $\mathrm{GMM}^{\prime}$ model with $m_{3 / 2}=20 \mathrm{TeV}$.

arbitrarily large soft terms are statistically favored-in fact, they are all bounded from above since once they get too big, they will lead either to nonstandard EW vacua or else too large a value of $m_{Z}^{\mathrm{PU}}$. In this way, models such as split SUSY or high scale SUSY would be ruled out since for a fixed (natural) value of $\mu$ (which is not then available for fine-tuning), they would necessarily lead to $m_{Z}^{\mathrm{PU}} \gg(2-5) m_{Z}^{\mathrm{OU}}$.
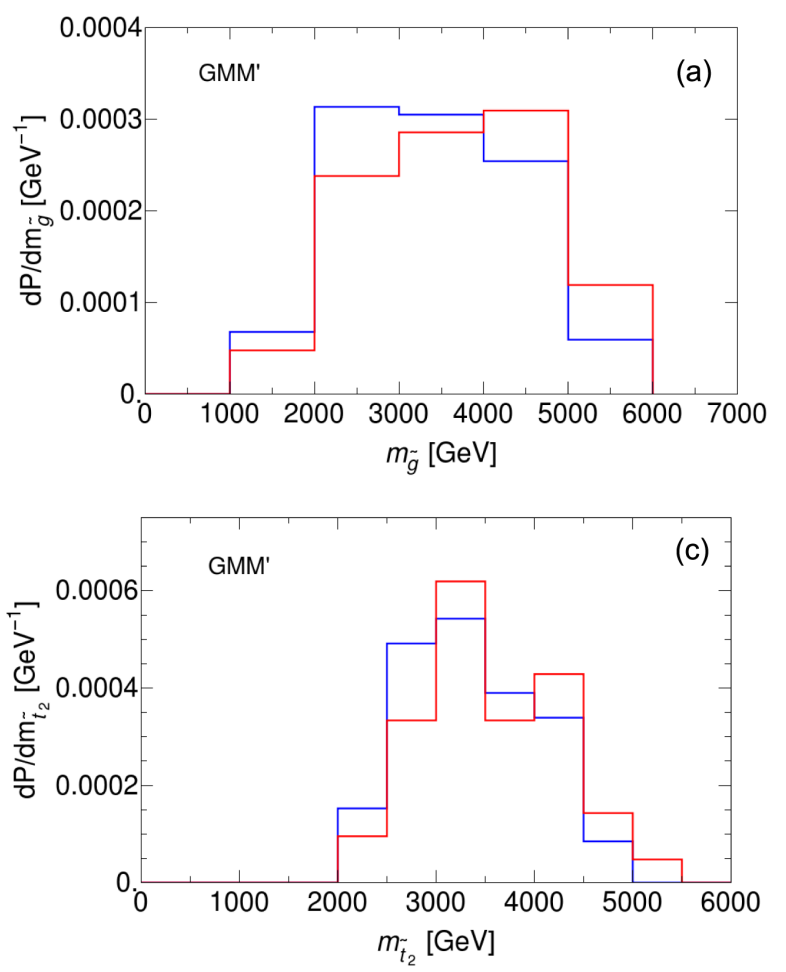

\section{Higgs and sparticle mass distributions for varying $\boldsymbol{n}_{\mathbf{0}}$}

In Fig. 3, we show the probability distribution for the light Higgs mass $d P / d m_{h}$ vs $m_{h}$ from our general landscape scans using $n_{1 / 2}=1$ but with $n_{0}=1$ (blue line) and 2 (red line). Both distributions peak around $m_{h} \sim 125 \mathrm{GeV}$, but the general scan with the harder $n_{0}=2$ statistical draw on scalar and trilinear soft terms is more sharply peaked around $125 \mathrm{GeV}$ than the $n_{0}=1$ case. This confirms the behavior shown previously in Fig. 2 for the more restrictive scan. We also generated scans with $n_{0}=3$ and 4 , but these tend to become very inefficient since as $n_{0}$ increases, one gets pushed almost always into no EWSB or CCB minima, or minima with too large a value of $m_{\text {weak }}^{\mathrm{PU}}$.

In Fig. 4, we show probability distributions for (a) $d P / d m_{\tilde{g}}$ vs $m_{\tilde{g}}$, (b) $d P / d m_{\tilde{t}_{1}}$ vs $m_{\tilde{t}_{1}}$, (c) $d P / d m_{\tilde{t}_{2}}$ vs $m_{\tilde{t}_{2}}$, and (d) $d P / d m_{A}$ vs $m_{A}$. From frame 4(a), we see that the landscape prediction for $m_{\tilde{g}}$ lies between 1.5 and $5 \mathrm{TeV}$ with a peak around $2.5 \mathrm{TeV}$ for $n_{0}=1$ and around $4.5 \mathrm{TeV}$ for $n_{0}=2$. Thus, contrary to traditional naturalness, stringy naturalness predicts a gluino mass typically well above LHC mass limits. The reach of HE-LHC with $\sqrt{s}=$ $27 \mathrm{TeV}$ has been computed in Ref. [95] where the 95\% C.L. LHC reach with $15 \mathrm{ab}^{-1}$ was found to be $m_{\tilde{g}} \lesssim 6 \mathrm{TeV}$. This is to be compared with the $(5 \sigma)$ reach of HL-LHC with $3 \mathrm{ab}^{-1}$ which extends to $m_{\tilde{g}} \sim 2.8 \mathrm{TeV}$ [96]. Thus, an energy doubling of LHC may well be required to discover SUSY in the $p p \rightarrow \tilde{g} \tilde{g} X$ channel.
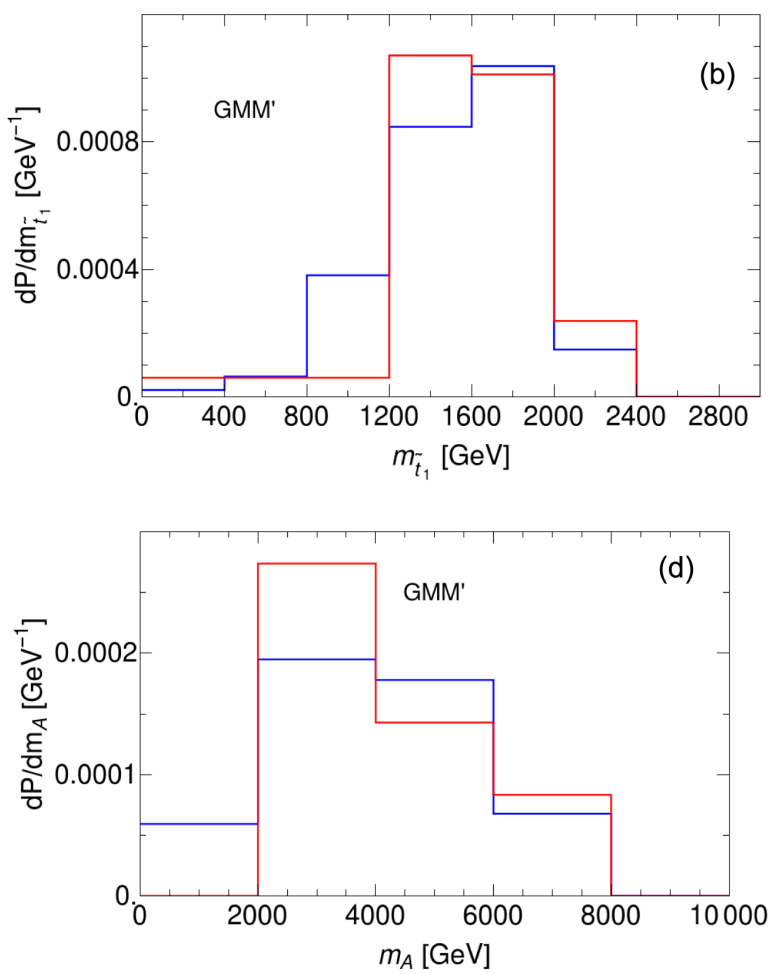

FIG. 4. Upper panels: Distributions in $m_{\tilde{g}}$ (left) and $m_{\tilde{t}_{1}}$ (right). Lower panels: Distributions in $m_{\tilde{t}_{2}}$ (left) and $m_{A}$ (right). Here, $n_{1 / 2}=1$ but $n_{0}=1$ (blue lines) and $n_{0}=2$ (red lines) are from statistical scans over the nGMM' model with $m_{3 / 2}=20 \mathrm{TeV}$. 
The distributions for $m_{\tilde{g}}$ change little with varying $n_{0}$ since the gaugino mass distribution depends instead on $n_{1 / 2}$.

In frame $4(\mathrm{~b})$, the landscape probability distribution for $m_{\tilde{t}_{1}}$ lies between $m_{\tilde{t}_{1}}: 1-2 \mathrm{TeV}$ with a peak probability around $m_{\tilde{t}_{1}} \sim 1.5 \mathrm{TeV}$ for both cases $n_{0}=1$ (blue line) and $n_{0}=2$ (red line). These distributions hardly depend on the $n_{0}$ value since for fixed $\mu \sim m_{\text {weak }}$, the largest contribution to $m_{\text {weak }}$ typically comes from $\Sigma_{u}^{u}\left(\tilde{t}_{1,2}\right)$ which sets the upper bound on $m_{\tilde{t}_{1}}$. The current limit from LHC Run 2 is that $m_{\tilde{t}_{1}} \gtrsim 1.1 \mathrm{TeV}[97,98]$. Thus, we see that LHC Run 2 has only started exploring the predicted stringy natural parameter space via stop pair production. For comparison, the $5 \sigma$ (95\% C.L.) HE-LHC reach with $15 \mathrm{ab}^{-1}$ extends to stop masses of 3 (3.5) TeV. Thus, again we would require an approximate doubling of LHC energy in order to cover the entire range of stop masses in landscape SUSY.

In frame $4(\mathrm{c})$, we see the landscape prediction for $m_{\tilde{t}_{2}}$ lies in the $2-5 \mathrm{TeV}$ range. The reach of HL- and HE-LHC for $\tilde{t}_{2}$ should be similar to their reaches for $m_{\tilde{t}_{1}}$. Thus, we would expect HE-LHC to cover only about half the expected mass range for the heavier top-squark $\tilde{t}_{2}$. The predicted statistical distribution for $m_{\tilde{t}_{2}}$ shifts to higher $m_{\tilde{t}_{2}}$ values for larger $n_{0}$ as might be expected.

In frame 4(d), we find the distribution for $m_{A}$ to lie within the $m_{A} \sim 1-8 \mathrm{TeV}$ range with a peak around $m_{A} \sim 3 \mathrm{TeV}$ for both $n_{0}=1$ and $n_{0}=2$. The upper bound on $m_{A}$ comes from the $m_{H_{d}}^{2} /\left(\tan ^{2} \beta-1\right)$ term in Eq. (2): if it is too large, then $m_{\text {weak }}^{\mathrm{PU}}$ will become too large. From this point of view, it is not surprising that the Higgs sector looks highly SM-like at LHC so far since there is a decoupling of heavier Higgs particles embedded mainly in the $H_{d}$ multiplet while the $H_{u}$ multiplet is very SM-like.

In Fig. 5, we show the string landscape prediction for first/second generation matter scalars, as typified by $m_{\tilde{u}_{L}}$. From this plot, for $n_{1 / 2}=1$ and $n_{0}=1$, we see that first/ second generation matter scalars extend from 10 to $35 \mathrm{TeV}$ with a peak distribution around $m_{\tilde{u}_{L}} \sim 22 \mathrm{TeV}$. The upper

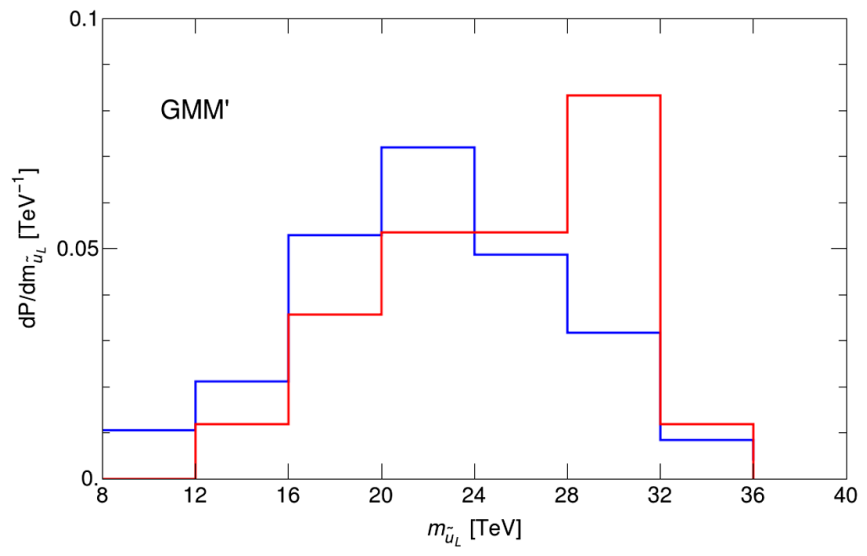

FIG. 5. Probability distribution $d P / d m_{\tilde{u}_{L}}$ vs $m_{\tilde{u}_{L}}$ from general scan for $n_{1 / 2}=1$ but for $n_{0}=1$ and 2 . bound on first/second generation matter scalars arises not from Yukawa terms (tiny) or $D$-terms (which largely cancel) but from two-loop RGE contributions which, if they get too large can drive top-squark soft terms to tachyonic values. As we increase $n_{0}$ to 2 , the distribution in $m_{\tilde{u}_{L}}$ hardens even further to a peak around $m_{\tilde{u}_{L}} \sim 30 \mathrm{TeV}$. Both first and second generation matter scalars are pulled to a common upper bound since the twoloop RGE terms are flavor independent. This leads to the string landscape mixed quasi-degeneracy/decoupling solution to the SUSY flavor and $C P$ problems [19]. In fact, in previous times model builders fought a hard battle to find schemes which lead to universal scalar masses as a means to solve the SUSY flavor problem. In contrast, in the string landscape picture, the expected nonuniversality of scalar masses turns out to be an asset since the different soft terms can be drawn to sufficiently large values while their contributions to the weak scale remain small. This mechanism leads to its own mixed quasi-degeneracy/decoupling solution to the SUSY flavor and $C P$ problems.

\section{CONCLUSIONS}

In this paper, our main goal was to examine the form of soft SUSY breaking terms that would arise in string compactifications to a $4 \mathrm{D}, N=1$ supergravity theory including the MSSM as the low energy EFT (the fertile patch). The statistical approach adopted here-pioneered by Weinberg, Bousso and Polchinski, Susskind, Douglas $[2,4,5,14]$, and others - does not depend on any particular string theory vacuum, but rather on the existence of a vast landscape of metastable string vacua where each may correspond to a different pocket universe (with different 4D laws of physics) within the multiverse. In string theory, one expects of order ten hidden sectors per vacuum solution, and several of these may contribute to the overall SUSY breaking scale. Since nothing in string theory prefers one SUSY breaking VEV over another, it is commonly expected that these will be distributed uniformly in the landscape. In such a case, there is a statistical draw to large soft terms since most of the volume of the multidimensional SUSY breaking space resides on the outermost shells $[8,14,15]$. The draw to large soft terms must be tempered by the requirement that the derived value of the pocketuniverse weak scale lie not too far removed from the measured value in our universe, lest atoms as we understand them not arise (the atomic principle) [20,21]. Our methodology here is rather modest: we merely combine these two ideas and then apply them to a statistical evaluation of expected soft terms arising in the MSSM.

We assumed the EFT consisted of the usual MSSM visible sector fields along with a hidden sector of moduli fields which would serve as the arena for SUSY breaking.

\footnotetext{
${ }^{5}$ See also Ref. [99].
} 
Using the well-known formulas for soft SUSY breaking terms in $N=1$ SUGRA, we would expect the gaugino masses $m_{1 / 2}$, the various scalar masses $m_{0}(i), m_{H_{u, d}}$, and the $A$ terms to scan independently due to their different functional dependence on the moduli fields.

For the soft breaking scalar masses, we expect generally nonuniversal soft terms due to different dependence of the Kähler metric on the compactified space. This reflects the expected geography of visible sector fields on the compactified manifold, as emphasized by Nilles and Vaudrevange [100] (whose conclusions were drawn from the context of heterotic orbifold models). In past times, nonuniversality of soft scalar masses was a thing to be avoided in that it could lead to dangerous flavor-violating processes. Various contorted model-building efforts were thus made to avoid the generic nonuniversality expected from realistic string compactifications. However, in the context of the string landscape, scalar mass nonuniversality turns out to be a desired property. This is because the landscape likely contains a statistical draw toward large soft terms, especially in the scalar mass sector. The draw to large $m_{H_{u}}^{2}$, which stops just short of the living dangerously feature of "no-EWSB," pulls $m_{H_{u}}^{2}$ to values associated with radiatively driven naturalness, wherein large high scale soft terms are evolved via RGEs to natural values at the weak scale [17]. Likewise, $A$ terms are drawn large enough to generate maximal mixing in the stop sector, thus minimizing the top-squark contributions to the weak scale while lifting $m_{h} \rightarrow 125 \mathrm{GeV}$, while stopping short of such large values as to generate CCB minima in the scalar potential $[17,18]$. Also, first/second generation scalars are drawn to a common upper bound in the $20-40 \mathrm{TeV}$ range which leads to a mixed quasi-degeneracy/decoupling solution to the SUSY flavor and $C P$ problems [19].

We also examined the soft terms in the context of how strongly they would be statistically drawn to large values by the string landscape. In many viable string models, the tree level gauge kinetic function depends only on the dilaton field so that a statistical pull of $m$ (gaugino $)^{n_{1 / 2}}$ with $n_{1 / 2}=1$ is expected. In contrast, the scalar masses and $A$ terms typically depend on all the moduli fields which would contribute to SUSY breaking, and thus a much stronger draw of $m_{\text {soft }}^{n_{0}}$ with $n_{0} \gg 1$ may be expected.

We illustrated the consequences of these different statistical draws in our scans over generalized mirage-mediation model GMM' parameter space wherein comparable modulimediated and anomaly-mediated contributions to soft terms arise. The cases with $n_{0}>n_{1 / 2}$ lead to predictions of greater splitting in the SUSY particle mass spectrum with first/
TABLE I. Expected range of Higgs and sparticle masses in the generalized mirage mediation $\left(\mathrm{GMM}^{\prime}\right)$ model from the string landscape with $n_{1 / 2}=1$ but with $n_{0}=1$ or $n_{0}=2$.

\begin{tabular}{lcc}
\hline \hline Mass & $n_{0}=1$ & $n_{0}=2$ \\
\hline$m_{h}$ & $125_{-4}^{+1} \mathrm{GeV}$ & $125_{-4}^{+1} \mathrm{GeV}$ \\
$m_{\tilde{g}}$ & $3.5 \pm 2.5 \mathrm{TeV}$ & $4 \pm 2 \mathrm{TeV}$ \\
$m_{\tilde{t}_{1}}$ & $1.6 \pm 0.8 \mathrm{TeV}$ & $1.6 \pm 0.8 \mathrm{TeV}$ \\
$m_{\tilde{t}_{2}}$ & $3.5 \pm 1.5 \mathrm{TeV}$ & $3.5 \pm 1.5 \mathrm{TeV}$ \\
$m_{A}$ & $4 \pm 2 \mathrm{TeV}$ & $4 \pm 2 \mathrm{TeV}$ \\
$m_{\tilde{f}}(1,2)$ & $22 \pm 10 \mathrm{TeV}$ & $30_{-18}^{+6} \mathrm{TeV}$ \\
\hline \hline
\end{tabular}

second generation scalar masses $\gg$ third generation and gaugino masses. As $n_{0}$ increases relative to $n_{1 / 2}$, the Higgs mass probability distribution sharpens even more to its expected peak at $m_{h} \sim 125 \mathrm{GeV}$.

What are the phenomenological consequences of the string landscape for LHC and dark matter searches? Our results are summarized in Table I from our scans over the $\mathrm{GMM}^{\prime}$ model with $n_{1 / 2}=1$ and with $n_{0}=1$ or 2 . Typically, our statistical landscape approach to SUSY phenomenology predicts a Higgs mass $m_{h} \simeq 125 \mathrm{GeV}$ with sparticle masses beyond LHC reach. Since the landscape predicts $m_{\tilde{g}} \sim 3.5 \pm$ $2.5 \mathrm{TeV}$ and $m_{\tilde{t}_{1}} \sim 1.6 \pm 0.8 \mathrm{TeV}$, an energy upgrade of LHC to at least $\sqrt{s} \sim 27 \mathrm{TeV}$ may be needed for SUSY discovery in the gluino pair or top-squark pair production channels. However, since the Higgsino mass parameter $\mu$ is required not-to-far from $m_{\text {weak }} \sim 100 \mathrm{GeV}$, it might be possible for LHC experiments to eke out a signal from direct Higgsino pair production reactions such as $p p \rightarrow \tilde{\chi}_{1}^{0} \tilde{\chi}_{2}^{0}$ in the soft, opposite-sign dilepton channel [23], perhaps in association with a hard jet radiation [101-104]. The parameter space for this SUSY discovery channel is just beginning to be explored [105].

Regarding dark matter, we would expect it to be composed of both SUSY DFSZ axions [66,106] (which have a suppressed coupling to photons [107]) along with a smaller component $(\sim 10 \%-20 \%)$ of Higgsino-like WIMPs [108]. The multiton noble liquid detectors now being deployed should have future sensitivity to the entire expected parameter space [109], so we would expect a WIMP discovery should still be forthcoming in the next 5-10 years.

\section{ACKNOWLEDGMENTS}

This material is based upon work supported by the U.S. Department of Energy, Office of Science, Office of High Energy Physics under Award No. DE-SC-0009956. 
[1] G. 't Hooft, Naturalness, chiral symmetry, and spontaneous chiral symmetry breaking, NATO Sci. Ser. B 59, 135 (1980).

[2] S. Weinberg, Anthropic Bound on the Cosmological Constant, Phys. Rev. Lett. 59, 2607 (1987).

[3] S. Weinberg, The cosmological constant problem, Rev. Mod. Phys. 61, 1 (1989).

[4] R. Bousso and J. Polchinski, Quantization of four form fluxes and dynamical neutralization of the cosmological constant, J. High Energy Phys. 06 (2000) 006.

[5] L. Susskind, The anthropic landscape of string theory arXiv:hep-th/0302219.

[6] F. Denef and M. R. Douglas, Distributions of flux vacua, J. High Energy Phys. 05 (2004) 072.

[7] K. R. Dienes, The string Landscape: A personal perspective, in Perspectives on String Phenomenology (World Scientifc, Singapore, 2015), Vol. 22, pp. 81-115.

[8] N. Arkani-Hamed, S. Dimopoulos, and S. Kachru, Predictive landscapes and new physics at a TeV, arXiv: hep-th/0501082.

[9] M. K. Gaillard and B. W. Lee, Rare decay modes of the $k$ mesons in gauge theories, Phys. Rev. D 10, 897 (1974).

[10] M. R. Douglas, Basic results in vacuum statistics, C.R. Phys. 5, 965 (2004).

[11] H. Baer, V. Barger, and S. Salam, Naturalness versus stringy naturalness with implications for collider and dark matter searches, Phys. Rev. Research 1, 023001 (2019).

[12] K. J. Bae, H. Baer, V. Barger, and D. Sengupta, Revisiting the susy $\mu$ problem and its solutions in the LHC era, Phys. Rev. D 99, 115027 (2019).

[13] H. Baer, V. Barger, P. Huang, D. Mickelson, A. Mustafayev, and X. Tata, Radiative natural supersymmetry: Reconciling electroweak fine-tuning and the Higgs boson mass, Phys. Rev. D 87, 115028 (2013).

[14] M. R. Douglas, Statistical analysis of the supersymmetry breaking scale, arXiv:hep-th/0405279.

[15] L. Susskind, Supersymmetry breaking in the anthropic landscape, in From Fields to Strings: Circumnavigating Theoretical Physics (World Scientific, Singapore, 2004), pp. 1745-1749.

[16] H. Baer, V. Barger, H. Serce, and K. Sinha, Higgs and superparticle mass predictions from the landscape, J. High Energy Phys. 03 (2018) 002.

[17] H. Baer, V. Barger, M. Savoy, and H. Serce, The Higgs mass and natural supersymmetric spectrum from the landscape, Phys. Lett. B 758, 113 (2016).

[18] H. Baer, V. Barger, P. Huang, A. Mustafayev, and X. Tata, Radiative Natural Supersymmetry with a $125 \mathrm{GeV}$ Higgs Boson, Phys. Rev. Lett. 109, 161802 (2012).

[19] H. Baer, V. Barger, and D. Sengupta, Landscape solution to the susy flavor and $c p$ problems, Phys. Rev. Research 1, 033179 (2019).

[20] V. Agrawal, S. M. Barr, J. F. Donoghue, and D. Seckel, Viable range of the mass scale of the standard model, Phys. Rev. D 57, 5480 (1998).

[21] V. Agrawal, S. M. Barr, J. F. Donoghue, and D. Seckel, Anthropic Considerations in Multiple-Domain Theories and the Scale of Electroweak Symmetry Breaking, Phys. Rev. Lett. 80, 1822 (1998).
[22] H. Baer, V. Barger, and D. Sengupta, Mirage mediation from the landscape, Phys. Rev. Research 2, 013346 (2020).

[23] H. Baer, V. Barger, and P. Huang, Hidden susy at the LHC: The light higgsino-world scenario and the role of a lepton collider, J. High Energy Phys. 11 (2011) 031.

[24] H. Baer, V. Barger, H. Serce, and X. Tata, Natural generalized mirage mediation, Phys. Rev. D 94, 115017 (2016).

[25] W. Taylor and Y.-N. Wang, The F-theory geometry with most flux vacua, J. High Energy Phys. 12 (2015) 164.

[26] F. Denef, M. R. Douglas, and S. Kachru, Physics of string flux compactifications, Annu. Rev. Nucl. Part. Sci. 57, 119 (2007).

[27] M. R. Douglas, The Statistics of string M theory vacua, J. High Energy Phys. 05 (2003) 046.

[28] M. R. Douglas, The string theory landscape, Universe 5, 176 (2019).

[29] I. Broeckel, M. Cicoli, A. Maharana, K. Singh, and K. Sinha, Moduli stabilisation and the statistics of SUSY breaking in the landscape, arXiv:2007.04327.

[30] S. Kachru, R. Kallosh, A. Linde, and S. P. Trivedi, de sitter vacua in string theory, Phys. Rev. D 68, 046005 (2003).

[31] M. Cicoli, J. P. Conlon, and F. Quevedo, General analysis of large volume scenarios with string loop moduli stabilisation, J. High Energy Phys. 10 (2008) 105.

[32] R. Kallosh, A. Linde, E. McDonough, and M. Scalisi, dS Vacua and the Swampland, J. High Energy Phys. 03 (2019) 134.

[33] V.S. Kaplunovsky and J. Louis, Model-independent analysis of soft terms in effective supergravity and in string theory, Phys. Lett. B 306, 269 (1993).

[34] A. Brignole, Towards a theory of soft terms for the supersymmetric standard model, Nucl. Phys. B422, 125171 (1994).

[35] V. Balasubramanian, P. Berglund, J. P. Conlon, and F. Quevedo, Systematics of moduli stabilisation in CalabiYau flux compactifications, J. High Energy Phys. 03 (2005) 007.

[36] S. K. Soni and H. Weldon, Analysis of the supersymmetry breaking induced by $N=1$ supergravity theories, Phys. Lett. 126B, 215 (1983).

[37] E. Cremmer, S. Ferrara, L. Girardello, and A. Van Proeyen, Yang-Mills theories with local supersymmetry: Lagrangian, transformation laws and SuperHiggs effect, Nucl. Phys. B212, 413 (1983).

[38] K. Choi, A. Falkowski, H. Nilles, and M. Olechowski, Soft supersymmetry breaking in kklt flux compactification, Nucl. Phys. B718, 113133 (2005).

[39] K. R. Dienes and C. F. Kolda, Twenty open questions in supersymmetric particle physics, arXiv:hep-ph/9712322.

[40] O. Lebedev, H. P. Nilles, S. Raby, S. Ramos-Snchez, M. Ratz, P. K. Vaudrevange, and A. Wingerter, A minilandscape of exact mssm spectra in heterotic orbifolds, Phys. Lett. B 645, 88 (2007).

[41] W. Buchmuller, K. Hamaguchi, O. Lebedev, and M. Ratz, Local grand unification, in GUSTAVOFEST: Symposium in Honor of Gustavo C. Branco: CP Violation and the Flavor Puzzle, 2005 (DESY, Hamburg, 2005), pp. 143156.

[42] M. Ratz, Notes on local grand unification, Soryushiron Kenkyu Electron. 116, A56 (2008). 
[43] L. Ibanez and G. G. Ross, $\mathrm{Su}(2) 1 \times \mathrm{u}(1)$ symmetry breaking as a radiative effect of supersymmetry breaking in guts, Phys. Lett. 110B, 215 (1982).

[44] K. Inoue, A. Kakuto, H. Komatsu, and S. Takeshita, Aspects of grand unified models with softly broken supersymmetry, Prog. Theor. Phys. 68, 927 (1982).

[45] K. Inoue, A. Kakuto, H. Komatsu, and S. Takeshita, Renormalization of supersymmetry breaking parameters revisited, Prog. Theor. Phys. 71, 413 (1984).

[46] L. E. Ibanez, Locally supersymmetric su (5) grand unification, Phys. Lett. 118B, 73 (1982).

[47] H. P. Nilles, M. Srednicki, and D. Wyler, Weak interaction breakdown induced by supergravity, Phys. Lett. 120B, 346 (1983).

[48] J. Ellis, J. S. Hagelin, D. Nanopoulos, and K. Tamvakis, Observable gravitationally induced baryon decay, Phys. Lett. 124B, 484 (1983).

[49] L. Alvarez-Gaume, J. Polchinski, and M. B. Wise, Minimal low-energy supergravity, Nucl. Phys. B221, 495 (1983).

[50] M. Dine, A. E. Nelson, Y. Nir, and Y. Shirman, New tools for low energy dynamical supersymmetry breaking, Phys. Rev. D 53, 2658 (1996).

[51] G. Giudice and R. Rattazzi, Theories with gauge-mediated supersymmetry breaking, Phys. Rep. 322, 419 (1999).

[52] L. Randall and R. Sundrum, Out of this world supersymmetry breaking, Nucl. Phys. B557, 79118 (1999).

[53] G. F. Giudice, R. Rattazzi, M. A. Luty, and H. Murayama, Gaugino mass without singlets, J. High Energy Phys. 12 (1998) 027.

[54] J. A. Bagger, T. Moroi, and E. Poppitz, Anomaly mediation in supergravity theories, J. High Energy Phys. 04 (2000) 009.

[55] P. Bintruy, M. K. Gaillard, and B. D. Nelson, One loop soft supersymmetry breaking terms in superstring effective theories, Nucl. Phys. B604, 3274 (2001).

[56] H. Baer, V. Barger, and D. Sengupta, Anomaly-mediated susy breaking model retrofitted for naturalness, Phys. Rev. D 98, 015039 (2018).

[57] A. Arbey, M. Battaglia, A. Djouadi, F. Mahmoudi, and J. Quevillon, Implications of a $125 \mathrm{gev}$ Higgs for supersymmetric models, Phys. Lett. B 708, 162 (2012).

[58] P. Draper, P. Meade, M. Reece, and D. Shih, Implications of a $125 \mathrm{GeV}$ higgs boson for the MSSM and low-scale supersymmetry breaking, Phys. Rev. D 85, 095007 (2012).

[59] H. Baer, V. Barger, and A. Mustafayev, Neutralino dark matter in msugra with a $125 \mathrm{GeV}$ light higgs scalar, J. High Energy Phys. 05 (2012) 091.

[60] H. Baer, V. Barger, and S. Salam, Naturalness versus stringy naturalness (with implications for collider and dark matter searches), Phys. Rev. Research 1, 023001 (2019).

[61] J. Casas and C. Munoz, A natural solution to the $\mu$ problem, Phys. Lett. B 306, 288 (1993).

[62] G. Giudice and A. Masiero, A natural solution to the $\mu$ problem in supergravity theories, Phys. Lett. B 206, 480 (1988).

[63] U. Ellwanger, C. Hugonie, and A. M. Teixeira, The nextto-minimal supersymmetric standard model, Phys. Rep. 496, 177 (2010).
[64] K. J. Bae, E. J. Chun, and S. H. Im, Cosmology of the dfsz2 axino, J. Cosmol. Astropart. Phys. 03 (2012) 013.

[65] K. J. Bae, H. Baer, and E. J. Chun, Mixed axion/neutralino dark matter in the SUSY dfsz2 axion model, J. Cosmol. Astropart. Phys. 12 (2013) 028.

[66] K. J. Bae, H. Baer, and E. J. Chun, Mixed axion/neutralino dark matter in the susy dfsz axion model, J. Cosmol. Astropart. Phys. 12 (2013) 028.

[67] M. Dine, W. Fischler, and M. Srednicki, A simple solution to the strong cp problem with a harmless axion, Phys. Lett. 104B, 199 (1981).

[68] A. Zhitnitsky, On possible suppression of the axion hadron interactions, (In Russian), Sov. J. Nucl. Phys. 31, 260 (1980).

[69] S. M. Barr and D. Seckel, Planck-scale corrections to axion models, Phys. Rev. D 46, 539 (1992).

[70] R. Holman, S. D. Hsu, T. W. Kephart, E. W. Kolb, R. Watkins, and L. M. Widrow, Solutions to the strong-cp problem in a world with gravity, Phys. Lett. B 282, 132 (1992).

[71] M. Kamionkowski and J. March-Russell, Planck scale physics and the Peccei-Quinn mechanism, Phys. Lett. B 282, 137 (1992).

[72] R. Kallosh, A. Linde, D. Linde, and L. Susskind, Gravity and global symmetries, Phys. Rev. D 52, 912935 (1995).

[73] K. Babu, I. Gogoladze, and K. Wang, Stabilizing the axion by discrete gauge symmetries, Phys. Lett. B 560, 214 (2003).

[74] H. M. Lee, S. Raby, M. Ratz, G. G. Ross, R. Schieren, K. Schmidt-Hoberg, and P. K. Vaudrevange, Discrete r symmetries for the mssm and its singlet extensions, Nucl. Phys. B850, 130 (2011).

[75] H. Baer, V. Barger, and D. Sengupta, Gravity safe, electroweak natural axionic solution to strong $\mathrm{cp}$ and susy $\mu$ problems, Phys. Lett. B 790, 58 (2019).

[76] H. Baer, V. Barger, D. Mickelson, A. Mustafayev, and X. Tata, Physics at a higgsino factory, J. High Energy Phys. 06 (2014) 172.

[77] H. Baer, M. Berggren, K. Fujii, J. List, S.-L. Lehtinen, T. Tanabe, and J. Yan, The ILC as a natural SUSY discovery machine and precision microscope: From light higgsinos to tests of unification, Phys. Rev. D 101, 095026 (2020).

[78] K. Choi, K.-S. Jeong, and K.-i. Okumura, Phenomenology of mixed modulus-anomaly mediation in fluxed string compactifications and brane models, J. High Energy Phys. 09 (2005) 039.

[79] A. Falkowski, O. Lebedev, and Y. Mambrini, Susy phenomenology of kklt flux compactifications, J. High Energy Phys. 11 (2005) 034.

[80] K. Choi, K. S. Jeong, T. Kobayashi, and K.-i. Okumura, Tev scale mirage mediation and natural little supersymmetric hierarchy, Phys. Rev. D 75, 095012 (2007).

[81] M. Endo, M. Yamaguchi, and K. Yoshioka, A bottom-up approach to moduli dynamics in heavy gravitino scenario: Superpotential, soft terms and sparticle mass spectrum, Phys. Rev. D 72, 015004 (2005).

[82] D. Matalliotakis and H. Nilles, Implications of nonuniversality of soft terms in supersyrnmetric grand unified theories, Nucl. Phys. B435, 115128 (1995). 
[83] M. Olechowski and S. Pokorski, Electroweak symmetry breaking with non-universal scalar soft terms and large tan solutions, Phys. Lett. B 344, 201 (1995).

[84] P. Nath and R. Arnowitt, Nonuniversal soft susy breaking and dark matter, Phys. Rev. D 56, 2820 (1997).

[85] J. Ellis, K. Olive, and Y. Santoso, The mssm parameter space with non-universal higgs masses, Phys. Lett. B 539, 107 (2002).

[86] J. Ellis, T. Falk, K. A. Olive, and Y. Santoso, Exploration of the mssm with non-universal higgs masses, Nucl. Phys. B652, 259347 (2003).

[87] H. Baer, A. Mustafayev, S. Profumo, A. Belyaev, and X. Tata, Direct, indirect and collider detection of neutralino dark matter in SUSY models with non-universal Higgs masses, J. High Energy Phys. 07 (2005) 065.

[88] F. E. Paige, S. D. Protopopescu, H. Baer, and X. Tata, ISAJET 7.69: A Monte Carlo event generator for $p p$, anti-p p, and e+e- reactions, arXiv:hep-ph/0312045.

[89] K. Uno, Search for squarks and gluinos in final states with jets and missing transverse momentum at $\sqrt{s}=13 \mathrm{TeV}$ using $139 \mathrm{fb}^{-1}$ data with the ATLAS detector, Proc. Sci., LeptonPhoton2019 (2019) 186.

[90] A. M. Sirunyan, A. Tumasyan, W. Adam, F. Ambrogi, T. Bergauer, J. Brandstetter, M. Dragicevic, J. Er, A. Escalante Del Valle et al., Search for supersymmetry in proton-proton collisions at 13 tev in final states with jets and missing transverse momentum, J. High Energy Phys. 10 (2019) 244.

[91] J. R. Ellis, K. Enqvist, D. V. Nanopoulos, and F. Zwirner, Observables in low-energy superstring models, Mod. Phys. Lett. A 01, 57 (1986).

[92] R. Barbieri and G. F. Giudice, Upper bounds on supersymmetric particle masses, Nucl. Phys. B306, 63 (1987).

[93] S. Dimopoulos and G. Giudice, Naturalness constraints in supersymmetric theories with non-universal soft terms, Phys. Lett. B 357, 573 (1995).

[94] G. W. Anderson and D. J. Castaño, Challenging weak-scale supersymmetry at colliders, Phys. Rev. D 53, 2403 (1996).

[95] H. Baer, V. Barger, J. S. Gainer, D. Sengupta, H. Serce, and $\mathrm{X}$. Tata, LHC luminosity and energy upgrades confront natural supersymmetry models, Phys. Rev. D 98, 07510 (2018).

[96] H. Baer, V. Barger, J. S. Gainer, P. Huang, M. Savoy, D. Sengupta, and X. Tata, Gluino reach and mass extraction at the LHC in radiatively-driven natural susy, Eur. Phys. J. C 77, 499 (2017).

[97] Search for new phenomena with top quark pairs in final states with one lepton, jets, and missing transverse momentum in $p p$ collisions at $\sqrt{s}=13 \mathrm{TeV}$ with the ATLAS detector.

[98] A. M. Sirunyan et al., Search for direct top squark pair production in events with one lepton, jets, and missing transverse momentum at $13 \mathrm{TeV}$ with the CMS experiment, J. High Energy Phys. 05 (2020) 032.

[99] E. Dudas, P. Lamba, and S. K. Vempati, Diluting susy flavour problem on the landscape, Phys. Lett. B 804, 135404 (2020).

[100] H. P. Nilles and P. K. S. Vaudrevange, Geography of fields in extra dimensions: String theory lessons for particle physics, Mod. Phys. Lett. A 30, 1530008 (2015).

[101] Z. Han, G. D. Kribs, A. Martin, and A. Menon, Hunting quasidegenerate Higgsinos, Phys. Rev. D 89, 075007 (2014).

[102] H. Baer, A. Mustafayev, and X. Tata, Monojet plus soft dilepton signal from light higgsino pair production at LHC14, Phys. Rev. D 90, 115007 (2014).

[103] C. Han, D. Kim, S. Munir, and M. Park, Accessing the core of naturalness, nearly degenerate higgsinos, at the LHC, J. High Energy Phys. 04 (2015) 132.

[104] H. Baer, V. Barger, M. Savoy, and X. Tata, Multichannel assault on natural supersymmetry at the high luminosity LHC, Phys. Rev. D 94, 035025 (2016).

[105] A. Canepa, T. Han, and X. Wang, The search for electroweakinos, Rev. Nucl. Part. Sci. 70 (2020), arXiv:2003.05450.

[106] K. J. Bae, H. Baer, and E. J. Chun, Mainly axion cold dark matter from natural supersymmetry, Phys. Rev. D 89, 031701(R) (2014).

[107] K. J. Bae, H. Baer, and H. Serce, Prospects for axion detection in natural susy with mixed axion-higgsino dark matter: Back to invisible?, J. Cosmol. Astropart. Phys. 06 (2017) 024.

[108] H. Baer, V. Barger, and D. Mickelson, Direct and indirect detection of Higgsino-like wimps: Concluding the story of electroweak naturalness, Phys. Lett. B 726, 330 (2013).

[109] H. Baer, V. Barger, and H. Serce, Susy under siege from direct and indirect detection experiments, Phys. Rev. D 94, 115019 (2016). 